\title{
Detection of diffuse radio emission at large distance from the center of the galaxy cluster A 2255
}

\author{
R. F. Pizzo ${ }^{1}$, A. G. de Bruyn ${ }^{1,2}$, L. Feretti ${ }^{3}$, and F. Govoni ${ }^{4}$ \\ 1 Kapteyn Institute, Postbus 800, 9700 AV Groningen, The Netherlands \\ e-mail: pizzo@astro.rug.nl \\ 2 ASTRON, Postbus 2, 7990 AA Dwingeloo, The Netherlands \\ Istituto di radioastronomia-CNR/INAF, via Gobetti 101, 40129 Bologna, Italy \\ 4 INAF-Osservatorio astronomico di Cagliari, Poggio dei Pini, Strada 54, 09012 Capoterra (CA), Italy
}

Received 21 December 2007 / Accepted 21 February 2008

\begin{abstract}
Aims. Low-frequency radio observations of galaxy clusters are the key to detecting the diffuse extended emission associated with them. The presence and properties of such radio sources in galaxy clusters reveal the existence of magnetic fields on a large scale and allow theories to be tested concerning both the origin of relativistic particles in the ICM and their propagation.

Methods. A deep radio observation of the A 2255 galaxy cluster was carried out at $85 \mathrm{~cm}$ with the WSRT. The good UV-coverage and sensitivity achieved by these observations allowed us to image the complex structure of the low-brightness, extended cluster sources (radio halo and relic).

Results. These sources show a larger extent than what has been imaged so far at this frequency, with two new structures located SW and NW of the cluster center and at projected distances of $2 \mathrm{Mpc}$ from it.

Conclusions. The physical properties of the newly detected structures, together with the active dynamical state of the cluster, support a connection with large-scale structure (LSS) formation shocks.
\end{abstract}

Key words. galaxies: clusters: general - galaxies: clusters: individual Abell 2255 - galaxies: intergalactic medium radio continuum: general

\section{Introduction}

The gravitational hierarchical formation of large-scale structures in the universe drives shocks in the intergalactic medium (IGM). These convert the kinetic energy associated with cosmic flows into thermal energy, with IGM temperatures reaching values of $5-10 \mathrm{keV}$ in massive clusters and $<1 \mathrm{keV}$ in large-scale filaments (e.g. Miniati 2002; Davé et al. 2001).

Shocks can re-accelerate old relativistic electron populations, released by the former AGN activity within a cluster, or they can also directly accelerate thermal electrons of the IGM. The detection of radio emission from intergalactic shocks has important implications for our understanding of cosmology and astrophysics: it provides a test of structure formation models, can confirm the existence of the undetected warm-hot intergalactic medium, and can trace its distribution (Keshet et al. 2004).

Cluster-wide relativistic electron populations are observed in several merging and post-merging clusters as diffuse steepspectrum structures. They are grouped in three classes: radio halos, relics, and mini-halos (Feretti \& Giovannini 1996). Radio halos are unpolarized, extended ( $\sim 1 \mathrm{Mpc}$ or more) structures located at the cluster center. Relics still have an extended shape, but they lie at the cluster periphery and show high polarization percentages ( 20\%). Mini-halos are detected around a powerful radio galaxy at the center of cooling core clusters and have a typical size of $\sim 500 \mathrm{kpc}$.

These diffuse cluster structures provide important information on the history and evolution of clusters, by improving our knowledge about the presence and importance of both large-scale magnetic fields and relativistic particles in the IGM. Furthermore, since up to now they have only been found in clusters with signatures of merging in the optical and X-ray domains, their detection could be considered to indicate a perturbed dynamical state.

The low radio-surface brightness and steep spectrum make the detection of halos and relics rather difficult. However, in the past few years several works on radio halos and their hosting clusters (e.g. Feretti 2003, 2005) have improved our knowledge of these radio sources. On the other hand, the location of relics in the outermost cluster regions makes their detection very problematic because, usually, only the cluster central regions are imaged at radio wavelengths with high sensitivity. Detailed broad band radio studies of relics are still missing; similarly, the comparison of radio with X-ray emission is not possible because of the lack of sensitivity of X-ray satellites in the peripheral cluster regions. A review of the current knowledge of relics is found in Giovannini \& Feretti (2004). One of the main goals of detecting "relic-like" structures in galaxy clusters is to constrain their origin.

The galaxy cluster A 2255 is nearby $(z=0.0806$, Struble $\&$ Rood 1999) and rich. ROSAT X-ray observations indicate that it has recently undergone a merger (Burns 1998; Feretti et al. 1997; Davis et al. 2003). Recent XMM-Newton observations of A 2255 revealed temperature asymmetries of the ICM and reached the conclusion that the merger happened $\sim 0.15$ Gyr ago, probably in the E-W direction with a still uncertain position angle (Sakelliou \& Ponman 2006). Optical studies of A 2255 reveal kinematical 
substructures in the form of several associated groups (Yuan et al. 2003), and the high ratio of velocity dispersion to X-ray temperature (6.3 keV; Horner 2001) also indicates a non-relaxed system. When studied at radio wavelengths, A 2255 shows a diffuse radio halo (located at the center of the cluster) and a relic source (at the cluster periphery), together with a large number of embedded head-tail radiogalaxies (Harris et al. 1980). This cluster is the first and only one in which polarized radio emission from a radio halo has been detected. The halo shows filaments of strong polarized emission ( $20-40 \%)$ with the magnetic fields fluctuating up to scales of $\sim 400 \mathrm{kpc}$ in size (Govoni et al. 2005).

We observed A 2255 at several radio wavelengths to better understand the nature of the polarized emission of the filaments in the halo (whether this is intrinsic or due to a projection effect) and to search for low surface-brightness features located far from the cluster center. We did not observe strong polarization from the halo filaments at $85 \mathrm{~cm}$, but the analysis is complicated by the presence of very strong Galactic polarization. These polarization results, as well as those at 18,21, and $25 \mathrm{~cm}$, will be discussed in a future paper (Pizzo et al., in prep.). In this Letter we present the recent results of the total intensity imaging of A 2255 at $85 \mathrm{~cm}$.

Throughout this paper we assume a $\Lambda \mathrm{CDM}$ cosmology with $H_{0}=71 \mathrm{~km} \mathrm{~s}^{-1} \mathrm{Mpc}^{-1}, \Omega_{\mathrm{m}}=0.3$, and $\Omega_{\Lambda}=0.7$. At the distance of A 2255, 1 " corresponds to $1.5 \mathrm{kpc}$ and $1^{\circ}=5.4 \mathrm{Mpc}$.

\section{Observations and reduction}

The observations were conducted with the Westerbork Synthesis Radio Telescope (WSRT) at $85 \mathrm{~cm}$. The array consists of fourteen $25 \mathrm{~m}$ dishes on an east-west baseline and uses earth rotation to fully synthesize the UV-plane. Ten of the telescopes are on fixed mountings, $144 \mathrm{~m}$ apart; the four $(2 \times 2)$ remaining dishes are movable along two rail-tracks. In the array, the baselines can extend from $36 \mathrm{~m}$ to $2.7 \mathrm{~km}$.

The $6 \times 12 \mathrm{~h}$ technique has been used for the observations. For full imaging over the whole primary beam, six array configurations were used with the four movable telescopes stepped at $12 \mathrm{~m}$ increments (i.e. half the dish diameter) and the shortest spacing running from $36 \mathrm{~m}$ to $96 \mathrm{~m}$. This provides continuous UV-coverage with interferometer baselines ranging from 36 to $2760 \mathrm{~m}$. The pointing and phase center of the telescope was directed towards $(\mathrm{J} 2000.0): \mathrm{RA}=17^{\mathrm{h}} 13^{\mathrm{m}} 00^{\mathrm{s}}$, Dec $=+64^{\circ} 07^{\prime} 59^{\prime \prime}$, which is located in the middle of A 2255.

The feeds/receivers cover the frequency range of about 310-380 MHz. The band can be completely covered by the new wide band correlator, which can independently process 8 tunable bands of $10 \mathrm{MHz}$ centered at 315, 324, 332, 341, 350, 359, 367, and $376 \mathrm{MHz}$. Each band is covered by 128 channels in 4 crosscorrelations to recover all Stokes parameters. For our observations, the size of the largest detectable structure is $\sim 1^{\circ}$, the size of the primary beam is $\sim 2^{\circ}$, and the resolution is $\sim 1^{\prime}$.

The data reduction was done with the WSRT-tailored NEWSTAR reduction package following the standard procedure: Fourier-Transform, Clean, and Restore. Self-calibration was applied to remove residual phase and gain variations. The data were flux-calibrated using 3C 295, for which we adopted a flux at $325 \mathrm{MHz}$ of $64.5 \mathrm{Jy}$. Automatic flagging was applied at the beginning to take care of bad data, and further flagging was done subsequently in an iterative way on the basis of the selfcal residuals. The total amount of flagged data was $\sim 25 \%$.

The final noise level in the image ranges from about $0.05 \mathrm{mJy} /$ beam to $0.15 \mathrm{mJy} /$ beam and is limited by classical confusion noise.

\section{Results}

The final $85 \mathrm{~cm}$ image of A 2255 is shown in Fig. 1. It shows the well known extended halo (located at the cluster center), the relic (located $10^{\prime}$ to the northeast from it) and the radio galaxies, at a very large distance from the center of the cluster: the Embryo $\left(\mathrm{RA}=15^{\mathrm{h}} 15^{\mathrm{m}} 05^{\mathrm{s}}\right.$, Dec $\left.=+64^{\circ} 03^{\prime} 42^{\prime \prime}\right)$ and the Beaver $(\mathrm{RA}=$ $17^{\mathrm{h}} 13^{\mathrm{m}} 19^{\mathrm{s}}$, Dec $\left.=+63^{\circ} 48^{\prime} 16^{\prime \prime}\right)$ lie at $\sim 1.6 \mathrm{Mpc}$ from the cluster center, while the Bean $\left(\mathrm{RA}=17^{\mathrm{h}} 15^{\mathrm{m}} 31^{\mathrm{s}}\right.$, Dec $\left.=+64^{\circ} 39^{\prime} 28^{\prime \prime}\right)$ lies at more than $3.5 \mathrm{Mpc}$ (quoted names are taken from Harris et al. 1980).

The $85 \mathrm{~cm}$ image improves over previous WSRT imaging (Feretti et al. 1997) by a factor of 20 in sensitivity and reveals a morphology of the extended emission of A 2255, that is much larger than the one known so far. At higher sensitivity, the central radio halo looks much more complex than in previous imaging, and it has a more extended shape ( $\sim 500 \mathrm{kpc}$ towards S and SW). Its southern region, where the Beaver radio galaxy lies, is directly connected with the tail of the Beaver radio galaxy, which has doubled in length to more than $1 \mathrm{Mpc}$ in the $85 \mathrm{~cm}$ image, compared to the $21 \mathrm{~cm}$ map (Pizzo \& de Bruyn 2007). Moreover, the already known northern relic shows a low brightness extent opposite to the cluster center, which was not visible at $21 \mathrm{~cm}$.

To understand the low-level, very extended emission, we made a low-resolution map (4'), where the point sources were removed. This image reveals an extended structure, which can be both positive and negative, on a scale of 0.5 to 1 degree, with a $T_{B} \sim 0.15 \mathrm{~K}$. We suspect this stems from structure in the Galactic foreground. The large-scale negative region that we note to the east of A 2255 is present in all 8 bands, so we suggest that is also related to the large-scale diffuse Galactic structure that is not properly imaged by the WSRT, whose shortest spacing is 36 meters.

Two new extended emission regions have now been detected. The new relics (we refer to them in this way) are one order of magnitude brighter than the "Galactic" structures discussed above. They are located NW (labeled A) and SW (labeled B) of the cluster center and lie at a projected distance of $2 \mathrm{Mpc}$ from it. Previous $21 \mathrm{~cm}$ imaging of the cluster (Pizzo \& de Bruyn 2007) revealed that these sources are genuine features and not a collection of discrete sources.

Another extended emission region seems to be present at low level at $\sim 500 \mathrm{kpc}$ westward of the new $\mathrm{SW}$ relic $(\sim \mathrm{RA}=$ $17^{\mathrm{h}} 08^{\mathrm{m}} 45^{\mathrm{s}}$, Dec $\left.=+63^{\circ} 43^{\prime} 45^{\prime \prime}\right)$. Moreover, a significant, extended feature is present also at the very eastern edge of the cluster. Its shape might suggest a physical association (jet structure) with the radio source WN B1717+6404 $\left(\mathrm{RA}=17^{\mathrm{h}} 17^{\mathrm{m}} 28^{\mathrm{s}}\right.$, Dec $\left.=+64^{\circ} 01^{\prime} 17^{\prime \prime}\right)$. Our new follow-up observations at the frequency range of $115-165 \mathrm{MHz}$ will tell us more about the nature of this extended feature.

The newly detected distant relics have different shapes. The SW relic appears like a filament about $8^{\prime}$ in length and $2^{\prime}$ in width. It has the same orientation of the known NE relic, but is located on the opposite side from the cluster center and at double the distance from it. The NW relic has a more complex morphology. We can distinguish 2 filaments (labels A1 and A2): A1 points towards the cluster center, and has a length of about $8^{\prime}$ and a width of about $1^{\prime}$; A2 is $\sim 13^{\prime}$ in length and $\sim 1^{\prime}$ in width and is perpendicular to $\mathrm{A} 1$. The $\mathrm{SW}$ relic has a total flux density of $\sim 17 \mathrm{mJy}$, the NW relic of $\sim 61 \mathrm{mJy}$.

We made low-resolution maps of the cluster at 320 and $380 \mathrm{MHz}$ and produced a spectral index map. The estimated values of the spectral index for the new relics are between $-2.5<\alpha<-0.5$, with the steeper values in the regions closer 


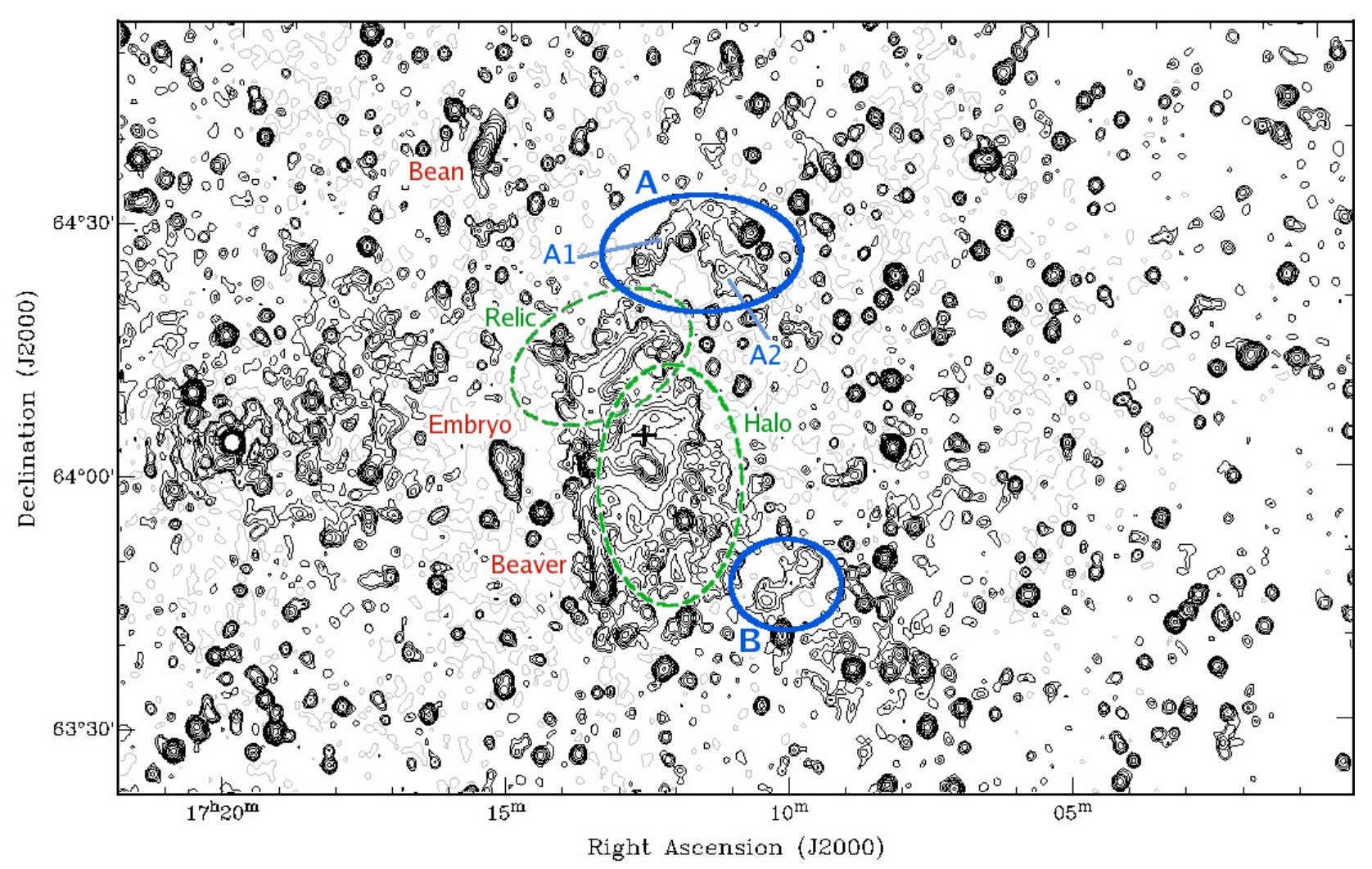

Fig. 1. Contour map of A 2255 at $85 \mathrm{~cm}$. The resolution $F W H M$ is $\sim 1^{\prime}$. The noise level is $\sim 0.1 \mathrm{mJy} /$ beam. The contours are $-0.3,0.3,0.6,1.2$, 2.4 , 4.8, 9.6, 20, 40, 80, $160 \mathrm{mJy} / \mathrm{beam}$. The cross indicates the cluster center. Please note that some of the emission surrounding the bright source $4 \mathrm{C}+64.21\left(\mathrm{RA}=17^{\mathrm{h}} 19^{\mathrm{m}} 58^{\mathrm{s}}, \mathrm{Dec}=+64^{\circ} 04^{\prime} 45^{\prime \prime}\right)$ is probably due to dynamic range limitations.

to the cluster center. The typical error $\sigma_{\alpha} \sim \pm 1$. A wider spectral range is needed to better determine the radio spectrum of these new features.

\section{Discussion}

Relics are found at the periphery of clusters both with and without a cooling core, suggesting that they may be related to minor or off-axis mergers, as well as to major mergers. The formation of relics is suggested to be related to shocks either by Fermi-I diffuse acceleration of ICM electrons (Enßlin et al. 1998; Keshet et al. 2004) or by adiabatic energization of the relativistic electrons confined in fossil radio plasma ("ghosts"), released by a former active radio galaxy (Enßlin \& Gopal-Krishna 2001). Shocks can be induced by the mergers of sub clusters ("merger shocks", Ricker \& Sarazin 2001), or they can be due to the accretion flows of IGM near the virial radius of the cluster ("accretion shocks" or "LSS shocks", Miniati 2003; Keshet et al. 2003).

Relic radio sources that can be associated with radio ghosts have been found in several clusters and a spectral index is also available for a few of them. The radio relic in Abell 85 (Slee et al. 2001) may be considered a prototype for this kind of objects, due to its size, morphology, and strong polarization.

If relics are directly produced by shocks, they should be found farther from the center of the clusters, where the X-ray surface brightness is very low. The ones associated with merger shocks should come in pairs and be located on the opposite side of the cluster center along the axis of the merger, with a ringlike structure elongated perpendicular to this direction (Ricker \& Sarazin 2001). This scenario is observed at radio wavelengths in the galaxy clusters A 3376 and A 3367, where a pair of large, optically unidentified, diffuse radio sources have been detected (Bagchi et al. 2006; Rottgering et al. 1997). This could also be the case for the previously known NE and the newly detected SW relics in A 2255, located on opposite sides with respect to the cluster center (although not symmetrically) and showing the same orientation, perpendicular to an NE-SW axis, that could describe the recently undergone merger. The origin of the extended ring-like features of A 3376 is probably due to merger shocks that accelerate the thermal electrons of the hot ICM or old relativistic particles to relativistic energies. On the other hand, in A 2255 the new relics lie at the virial radius of the cluster (Neumann 2005) and at a large projected distance from the X-ray emission associated with the cluster. This makes them more likely associated with shocks waves in the cosmic environment than with a hot ICM.

We note that the new relics of A 2255 are reminiscent of the filamentary structures of the halo detected by Govoni et al. (2005), which are elongated and organized in a kind of "net" or "web". This suggests that these structures could also be related to LSS or merger shocks and would naturally explain the high percentage of linear polarization.

The spectra of known LSS-related relics are steep $(\alpha \sim$ -1.2 ), and they are found to be linearly polarized at the level of $10 \%-30 \%$. The previously known NE relic shows such a polarization (Govoni et al. 2005), but we cannot confirm the presence of polarized flux for the newly detected structures so far. Using RM synthesis (Brentjens \& de Bruyn 2005), we have generated an RM-cube over a wide range of Faraday depths. It shows that most of the polarized emission in the field of A 2255 is due to 
the Galactic foreground, since it extends well beyond the cluster "boundary", and it has no counterpart in total intensity; however, there are several features in the RM-cube suggesting a link with continuum structures belonging to the cluster. Part of the NW relic may have been detected at a Faraday depth of about $-28 \mathrm{rad} / \mathrm{m}^{2}$, far from the depth of the bulk of the Galactic polarized emission (Pizzo et al., in prep.).

The spectral indices of the new relics $(-2.5<\alpha<-0.5$ with $\left.\sigma_{\alpha} \pm 1\right)$ seem to agree with the typical value of LSS shocks, although we should not expect a spectrum steeper than $\alpha \sim-1.5$ for LSS-related structures, since electrons are constantly accelerated at the shock front (priv. comm. with Matthias Hoeft). As the shock dissipates, the relativistic particles age, explaining the higher values of $\alpha$ we detect. Moreover, the trend shown by the spectral index, which is flatter at the periphery of the cluster and steepening towards the cluster center, is what one also expects for shock-related structures (see the relic sources in A 3667 , Roettiger et al. 1999). However, a wider spectral range is needed to confirm this result.

The morphology of the new structures, together with their size $(\simeq 1 \mathrm{Mpc}$ ), location (tangential with respect to the cluster center and at the virial radius of the cluster), spectra, and (possible) polarization support an LSS shock origin.

\section{Conclusions}

We have presented the results of new $85 \mathrm{~cm}$ WSRT observations of the galaxy cluster A 2255 .

- Our map reveals that the extended radio emission in A 2255 is both much more extended and complex than known before (Feretti et al. 1997).

- Two new extended emission regions are detected at a projected distance of $2 \mathrm{Mpc}$ from the cluster center.

- The spectral index of the new structures ranges between $-2.5<\alpha<-0.5$, with $\sigma_{\alpha} \pm 1$.

- The morphology of the new structures, together with their size ( $\simeq 1 \mathrm{Mpc}$ ), location (tangential with respect to the cluster center and at the virial radius of the cluster), spectra, and (possible) polarization support an LSS shock origin.

In the current models of large-scale structure formation, matter is distributed in filaments and sheets that are all connected. Radio emission is expected to be present in collapsing filaments, where the efficiency of electrons acceleration is higher than in cluster centers (Bagchi et al. 2002). We are now reaching the sensitivity needed for detecting radio emission from regions in between galaxy clusters. This is suggested by the discovery of elongated diffuse emission $(0917+75)$ at $\sim 3.8 \mathrm{Mpc}$ from the nearest rich cluster (Dewdney et al. 1991; Harris et al. 1993; Giovannini \& Feretti 2000), of a relic source in A 2069 (Giovannini et al. 1999) at $4.6 \mathrm{Mpc}$ from the cluster center and of extended diffuse radio emission coincident with the filament of galaxies $\mathrm{ZwCl} 2341.1+0000$, 2.5 Mpc in size (Bagchi et al. 2002). The steep spectrum of the diffuse inter-galactic radio sources and, based on current theoretical results (e.g. Miniati 2002), their connection with shocks in the IGM predict, at low radio frequencies, a sizable population of as yet undetected extended emissions around galaxy clusters. A recent ROSAT X-ray survey observation indicates that A 2255 belongs to the north ecliptic pole super cluster (Mullis et al. 2001). Therefore, it is possible that there are signs of interaction between A 2255 and several other galaxy clusters. A panoramic study of A 2255 out to many degrees ( 10 Mpc) is needed to search for such interaction. The detection of new filaments and relics related to LSS shocks will be enormously improved through observations with the next generation of lowfrequency radio telescopes like LOFAR, LWA, and SKA, for which we are preparing ourselves.

Acknowledgements. The Westerbork Synthesis Radio Telescope is operated by ASTRON (Netherlands Foundation for Research in Astronomy) with support from the Netherlands Foundation for Scientific Research (NWO).

\section{References}

Bagchi, J., Enßlin, T. A., Miniati, F., et al. 2002, New Astron., 7, 249 Bagchi, J., Durret, F., Neto, G. B. L., \& Paul, S. 2006, Science, 314, 791 Brentjens, M. A., \& de Bruyn, A. G. 2005, A\&A, 441, 1217 Burns, J. O. 1998, Science, 280, 400

Davé, R., Cen, R., Ostriker, J. P., et al. 2001, ApJ, 552, 473

Davis, D. S., Miller, N. A., \& Mushotzky, R. F. 2003, ApJ, 597, 202

Dewdney, P. E., Costain, C. H., McHardy, I., et al. 1991, ApJS, 76, 1055

Enßlin, T. A., Biermann, P. L., Klein, U., \& Kohle, S. 1998, A\&A, 332, 395

Enßlin, T. A., \& Gopal-Krishna. 2001, A\&A, 366, 26

Feretti, L. 2003, in Texas in Tuscany, XXI Symp. on Relativistic Astrophysics, ed. R. Bandiera, R. Maiolino, \& F. Mannucci, 209

Feretti, L. 2005, in X-Ray and Radio Connections, ed. L. O. Sjouwerman, \& K. K. Dyer, (Published electronically by NRAO,

http://www.aoc.nrao.edu/events/xraydio Held 3-6 February 2004 in Santa Fe, New Mexico, USA)

Feretti, L., Boehringer, H., Giovannini, G., \& Neumann, D. 1997, A\&A, 317, 432

Feretti, L., \& Giovannini, G. 1996, in Extragalactic Radio Sources, ed. R. D. Ekers, C. Fanti, \& L. Padrielli, IAU Symp., 175, 333

Giovannini, G., \& Feretti, L. 2000, New Astron., 5, 335

Giovannini, G., \& Feretti, L. 2004, J. Kor. Astron. Soc., 37, 323

Giovannini, G., Tordi, M., \& Feretti, L. 1999, New Astron., 4, 141

Govoni, F., Murgia, M., Feretti, L., et al. 2005, A\&A, 430, L5

Harris, D. E., Lari, C., Vallee, J. P., \& Wilson, A. S. 1980, A\&AS, 42, 319

Harris, D. E., Stern, C. P., Willis, A. G., \& Dewdney, P. E. 1993, AJ, 105, 769

Horner, D. 2001, Ph.D. Thesis

Keshet, U., Waxman, E., Loeb, A., Springel, V., \& Hernquist, L. 2003, ApJ, 585, 128

Keshet, U., Waxman, E., \& Loeb, A. 2004, New Astron. Rev., 48, 1119

Miniati, F. 2002, MNRAS, 337, 199

Miniati, F. 2003, MNRAS, 342, 1009

Mullis, C. R., Henry, J. P., Gioia, I. M., et al. 2001, ApJ, 553, L115

Neumann, D. M. 2005, A\&A, 439, 465

Pizzo, R. F., \& de Bruyn, G. 2007, in Extragalactic Jets: Theory and Observation from Radio to Gamma Ray, ed. T. A. Rector \& D. S. De Young

Ricker, P. M., \& Sarazin, C. L. 2001, ApJ, 561, 621

Roettiger, K., Burns, J. O., \& Stone, J. M. 1999, ApJ, 518, 603

Rottgering, H. J. A., Wieringa, M. H., Hunstead, R. W., \& Ekers, R. D. 1997, MNRAS, 290, 577

Sakelliou, I., \& Ponman, T. J. 2006, MNRAS, 367, 1409

Slee, O. B., Roy, A. L., Murgia, M., Andernach, H., \& Ehle, M. 2001, AJ, 122, 1172

Struble, M. F., \& Rood, H. J. 1999, ApJS, 125, 35

Yuan, Q., Zhou, X., \& Jiang, Z. 2003, VizieR Online Data Catalog, 214, 90053 